\title{
Welcome message from the Editors-in-Chief
}

\author{
Silvia Gabrielli ${ }^{1, *}$, Tiziana Catarci ${ }^{2}$ \\ ${ }^{1}$ Ubiquitous Interaction Group, CREATE-NET, via alla Cascata 56/D, I-38123 Povo di Trento, Italy; ${ }^{2}$ Dipartimento \\ di Informatica e Sistemistica, University of Rome "La Sapienza”, via Ariosto 25, I-00185 Rome, Italy
}

\begin{abstract}
Received on 5 October 2011
Copyright (C) 2011 Gabrielli and Catarci, licensed to ICST. This is an open access article distributed under the terms of the Creative Commons Attribution licence (http://creativecommons.org/licenses/by/3.0/), which permits unlimited use, distribution and reproduction in any medium so long as the original work is properly cited.
\end{abstract}

doi: 10.4108/trans.amsys.2011.el

On behalf of the Editorial board, we welcome you to the inaugural issue of the ICST Transactions on Ambient Systems!

We are delighted to launch this new transactions journal after a preparatory process that has received encouraging support from the Editorial Board and from ICST/ EAI.

The design of Ambient Systems is yielding novel challenges and opportunities to IT research and industry that require multidisciplinary, as well as visionary thinking. While electronics and sensors technology is becoming more and more invisible to the point of being woven in everyday environments, novel types of user experience and multimodal interaction need to be devised to provide fluid, intuitive and smart support to human activities. The main goal of this journal is to offer an open forum where innovative ideas, groundbreaking approaches and results can be promptly communicated and shared with the interested communities in order to advance the design of Ambient Systems. In particular, this transactions journal seeks contributions from the following areas:

- Pervasive Environments, Infrastructures and Applications design

- Immersive Media and Multimodal Interaction Design

- Embedded Systems and Software

- Mobile and Collaborative Computing

- Ambient Assisted Living

- Embodied agents and Affective Interaction

- Advanced and Wireless Networking solutions

- Distributed Healthcare and Edutainment

*Corresponding author. Email: silvia.gabrielli@create-net.org
- Multimodal systems for Sustainable Mobility

- Mixed and Augmented Reality

- Security, Privacy and Trust in Ambient Systems

- Smart Energy, Smart City solutions

- Calm and Persuasive Technology

- Context and Collective awareness, social sensing and activity recognition

- Brain-Computer Interaction

This inaugural issue presents five scientific contributions.

The first paper, by C. Di Ciccio et al. addresses the lack of interoperability and smart integration of domotic services for future homes. The authors present an innovative approach to service composition and the design of advanced user interfaces for smart homes, recently developed and tested within the SM4ALL European project.

The second paper, by E. Jafer et al. contributes to improving smart infrastructures for energy saving in buildings, by describing results collected during the design and deployment of a wireless sensor node platform for environmental monitoring and control of buildings.

The third paper, by A. Somov, provides a survey of approaches and methods for early wildfire detection in large territories by using wireless sensor networks, together with a comparative analysis of these methods in terms of their cost, power consumption and implementation complexity.

The fourth paper, by O.A. Postolache et al. describes recent research and prototype implementations of mobile healthcare systems for in home rehabilitation. These solutions aim to improve the management of patients in home rehabilitation by providing more accurate and up-to-date information, using pervasive computing of vital signs and motor activity records. 
Finally, the position paper, by J.C. Augusto, P.J. McCullagh and J.A. Augusto-Walkden, reflects on a number of safety issues presented by the wider deployment of Ambient Intelligence in daily life. In particular, it discusses possible negative consequences of system failure in critical application contexts, such as Ambient Assisted Living, by offering some viable solutions and design methods that should be adopted to avoid such risks.

For the preparation of this first issue we would like to acknowledge the work of all our Editors, reviewers and authors who have positively supported this publication initiative. We will be happy to receive from our readers any suggestions, including possible proposals for future special issues, which may contribute to further maintain the high scientific quality and relevance of this journal.

We hope you will find this first issue thought provoking for your research in the field of Ambient Systems and worth considering for your future dissemination work.

\section{About the Editors-in-Chief}

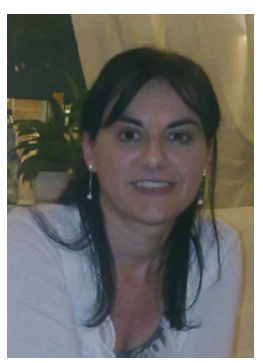

Silvia Gabrielli is Deputy Area Head of the Ubiquitous Interaction group at CREATE-NET (I) and adjunct Professor of Human-Computer Interaction at the University of Trento. Over the last ten years she has been a research scientist at FBKIRST (I), HCI-Lab (University of Udine), DIS (University of Rome La Sapienza), Interaction Design Institute Ivrea (I) and Interact Lab (University of Sussex, UK). She holds a Ph.D. in
Cognitive Sciences and a Master's degree in Psychology from the University of Padova (I). Her research focus is on the human-centered design of persuasive technology and multimodal interfaces for Personal Healthcare, Assisted Living and Eco-Sustainability.

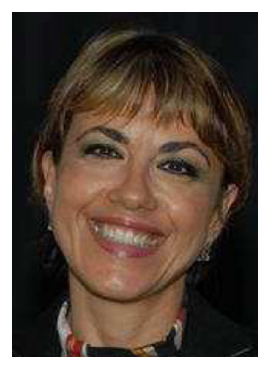

Tiziana Catarci got her Ph.D. in Computer Engineering in 1992 from the University of Roma "La Sapienza", Italy, where she is now a full Professor and Vice-Rector for ICT and Infrastructures. Her main research interests are in theoretical and application-oriented aspects of visual formalisms for databases, information visualization, database design, cooperative information systems, user interfaces, usability, digital libraries, data quality and Web access. On these topics she has published over 150 papers in leading journals and conferences and 20 books. Her contribution can be regarded as one of the first and most significant examples of deep analysis and formalization of the interaction between the user and the database, which takes in to consideration both usability issues and language-related aspects. With respect to applications, she has led or participated in various projects on visual query systems, usability studies and developed methodologies for database design. Particularly relevant are some European projects, including KIM, VENUS, FADIVA, LAURIN, SEWASIE, DELOS, Interop, WORKPAD, SM4All, SmartVortex, aiming at investigating different aspects of interface design and human-computer interaction. 